\title{
CONTROLE DE Brachiaria subquadripara E Brachiaria mutica Através de Diferentes Herbicidas Aplicados em Pós-emergênCia ${ }^{1}$
}

\author{
Control of Brachiaria subquadripara and Brachiaria mutica by Different \\ Herbicides Applied Post-emergence
}

CARBONARI, C.A. ${ }^{2}$, MARTINS, D. ${ }^{3}$ e TERRA, M.A. ${ }^{4}$

\begin{abstract}
RESUMO - Foram realizados dois experimentos, com objetivo de avaliar a suscetibilidade de Brachiaria subquadripara e Brachiaria mutica a diferentes herbicidas aplicados em pós-emergência. Os herbicidas e doses testados foram: glyphosate (Rodeo) a 2.400, 3.360 e 4.320 g e.a. ha ${ }^{-1}$ com Aterbane a 0,5\% v/v; glyphosate a 2.400, 3.360 e 4.320 g e.a. ha ${ }^{-1}$ com Silwet a $0,1 \% \mathrm{v} / \mathrm{v}$; imazapyr (Arsenal) a 750 e 1.500 g e.a. ha ${ }^{-1}$; e diquat (Reward) a 400 e 800 g i.a. ha ${ }^{-1}$ com aplicação seqüencial. Manteve-se uma testemunha sem aplicação de herbicidas. As parcelas experimentais foram constituídas de caixas d'água de $60 \times 60 \times$ $45 \mathrm{~cm}$, com $55 \mathrm{~L}$ de solo. A aplicação foi realizada quando as plantas se encontravam a $45 \mathrm{e}$ $65 \mathrm{~cm}$ de altura para B. subquadripara e B. mutica, respectivamente. Foi utilizado um pulverizador costal, a pressão constante de $\mathrm{CO}_{2}$ (2 bars), pontas $110.02 \mathrm{XR}$, com um consumo de calda de $200 \mathrm{~L} \mathrm{ha}^{-1}$. O controle foi avaliado visualmente, através de escala percentual de notas, além de se avaliar a massa seca das plantas. O herbicida glyphosate nas doses de 3.360 e 4.320 g e.a. ha $^{-1}$, independentemente do adjuvante testado, proporcionou controle excelente para as duas espécies, porém as parcelas que receberam glyphosate a 2.400 g e.a. ha ${ }^{-1}$ apresentaram controle apenas satisfatório. O herbicida imazapyr nas doses de 750 e 1.500 g e.a. ha ${ }^{-1}$ proporcionou controle eficiente para $B$. subquadripara e excelente para B. mutica. Já o herbicida diquat, apesar de duas aplicações e independentemente da dose utilizada, mostrou-se ineficiente no controle das duas espécies.
\end{abstract}

Palavras-chave: planta aquática, adjuvante, glyphosate, imazapyr, diquat.

\begin{abstract}
Two experiments were carried out to evaluate the susceptibility of Brachiaria subquadripara and Brachiaria mutica to different herbicides applied in post-emergence. The herbicides and doses used were: glyphosate (Rodeo) at 2,400, 3,360 and 4,320 (g a.e. ha ${ }^{-1}$ ) plus Aterbane 0.5\% v/v; glyphosate at 2,400, 3,360 and 4,320 (g a.e. ha $a^{-1}$ plus Silwet 0.1\% $v / v$; imazapyr (Arsenal) at 750 and 1,500 (g a.e. ha $^{-1}$ ), diquat (Reward) at 400 and 800 (g a.i. ha ${ }^{-1}$ ) with sequential application and an untreated control. The experimental plots were constituted of $60 \times 60 \times 45 \mathrm{~cm}$ water reservoirs, with $55 \mathrm{~L}$ of soil. Herbicides were applied when the plants reached 45 and $65 \mathrm{~cm}$ for B. subquadripara and B. mutica, respectively. A $\mathrm{CO}_{2}$ backpack sprayer was used with 2 bars, $110.02 \mathrm{XR}$ nozzles and consumption of $200 \mathrm{~L} \mathrm{ha}^{-1}$ spray solution. The control was visually evaluated, through percentile note scale, and plant dry mass as well. The herbicide glyphosate (doses of 3,360 and 4,320 $\mathrm{g}$ a.e. $\mathrm{ha}^{-1}$ ) showed an excellent control for the two species, regardless of the adjuvant tested. However, the plots that had received

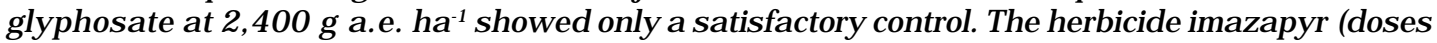
750 and $1,500 \mathrm{~g}$ a.e. $\mathrm{ha}^{-1}$ ) provided an effective control for B. subquadripara and a very effective control for B. mutica. The herbicide diquat, although applied twice and regardless of the dosage used, was found to be inefficient in controlling the two species.
\end{abstract}

Key words: aquatic plant, adjuvant, glyphosate, imazapyr, diquat.

Recebido para publicação em 9.9.2002 e na forma revisada em 27.10.2002.

Aluno de graduação, bolsista FAPESP, Departamento de Produção Vegetal da Faculdade de Ciências Agronômicas da Universidade Estadual Paulista - FCA-UNESP, Caixa postal 237, 18603-970 Botucatu-SP, <carbonari@ fca.unesp.br>; ${ }^{3}$ Professor Assistente, Doutor, Departamento de Produção Vegetal da FCA-UNESP; ${ }^{4}$ Eng.-Agr., M.S., Departamento de Produção Vegetal da FCA-UNESP. 


\section{INTRODUÇÃO}

Ambientes úmidos ou alagados, como várzeas e margem de corpos hídricos, proporcionam condições ecológicas favoráveis a uma grande proliferação de plantas daninhas, que surgem espontaneamente nessas condições. Dentre várias espécies, destacam-se Brachiaria subquadripara e Brachiaria mutica, pertencentes à família Poaceae, sendo originárias da África e introduzidas no Brasil como forrageiras, no entanto, acabaram tornando-se invasoras muito agressivas.

Essas espécies são conhecidas popularmente no Brasil por tanner-grass ( $B$. subquadripara) e capim-angola (B. mutica), sendo muito parecidas e freqüentemente confundidas quando ocorrem simultaneamente na mesma área. São muito abundantes em áreas úmidas e alagadas, resistindo muito bem a inundações temporárias - nessas condições apresentam apreciável massa verde, que fica flutuando sobre a água.

As duas espécies relatadas são plantas daninhas perenes e estoloníferas que se reproduzem facilmente por meios vegetativos, a partir de estolões que são formados em abundância, e produzem poucas sementes, as quais têm baixa eficiência de reprodução (Lorenzi, 1991; Kissmann, 1997; Roche et al., 1990). Apresentam-se como importantes invasoras da cultura do arroz irrigado por inundação e, principalmente, de canais de irrigação e drenagem (Kissmann, 1997). Também são encontradas em grandes quantidades nas margens de lagos e reservatórios utilizados para múltiplos usos da água, como geração de energia, recreação, pesca e navegação (Tanaka, 1998).

A ocorrência dessas plantas daninhas em lagos, represas e canais de irrigação proporciona elevada perda de água por transpiração, além de reduzir a velocidade da água nos canais, aumentando a sedimentação de partículas e encarecendo a manutenção destes (Oliveira Jr. \& Constantin, 2001). Pitelli (1998) afirma que estas espécies invadem represas rurais, facilitando o acúmulo de material erodido e reduzindo a capacidade de armazenamento de água e a própria vida útil do reservatório.

No Brasil, apesar do imenso potencial hídrico e dos grandes danos causados por essas plantas, são escassos os trabalhos visando o seu controle. Segundo Martins (1998), dentre os métodos de controle existentes e recomendados têm-se os herbicidas, sendo mundialmente mais utilizados os seguintes compostos: 2,4-D, glyphosate, diquat, endothal composto à base de cobre, fluridone e imazapyr.

Esta pesquisa teve como objetivo avaliar a eficiência de alguns herbicidas aplicados em pós-emergência, isolados ou acrescidos de adjuvantes, no controle de $B$. subquadripara e B. mutica.

\section{MATERIAL E MÉTODOS}

Os experimentos foram instalados e conduzidos no Núcleo de Pesquisas Avançadas em Matologia (NUPAM), pertencente ao Departamento de Produção Vegetal da Faculdade de Ciências Agronômicas/UNESP, campus de Botucatu-SP.

As plantas de B. subquadripara e B. mutica foram cultivadas em caixas d'água de fibra de vidro com as dimensões de $60 \times 60 \times 45 \mathrm{~cm}$, contendo cerca de 55 litros de solo de várzea, o que proporcionou profundidade de aproximadamente $15 \mathrm{~cm}$. As características químicas do solo utilizado nos experimentos foram: $\mathrm{pH}$ $\left(\mathrm{CaCl}_{2}\right)=5,1$; M.O. $\left(\mathrm{g} \mathrm{dm}^{-3}\right)=17 ; \mathrm{P}, \mathrm{B}, \mathrm{Cu}$, $\mathrm{Fe}, \mathrm{Mn}$ e $\mathrm{Zn}=64,0,12,7,8,319,25,4$ e $2,1 \mathrm{~g} \mathrm{dm}^{-3}$, respectivamente; $\mathrm{Al}, \mathrm{H}+\mathrm{Al}, \mathrm{K}, \mathrm{Ca}$, $\mathrm{Mg}, \mathrm{SB}$ e CTC $=1,34,2,2,45,28,75$ e 109, respectivamente. As características físicas eram: $60 \%$ de areia, $25 \%$ de argila e 9\% de silte. Foi realizada uma adubação de $80 \mathrm{~kg} \mathrm{ha}^{-1}$ da formulação comercial 8-28-16 antes do plantio e adubação nitrogenada de $80 \mathrm{~kg} \mathrm{ha}^{-1}$ com uréia 50 dias após o plantio.

As plantas foram reproduzidas vegetativamente, por meio de mudas, e podadas cerca de $20 \mathrm{~cm}$ acima do solo. Foram colocadas três mudas em cada caixa d'água, proporcionando assim boa distribuição das plantas. As plantas foram mantidas a pleno sol e com uma lâmina de água de 3 a $7 \mathrm{~cm}$ constantemente sobre o solo.

Os tratamentos utilizados nos dois experimentos foram: glyphosate (Rodeo) a $2.400,3.360$ e 4.320 g e.a. ha ${ }^{-1}$ com Aterbane a $0,5 \% \mathrm{v} / \mathrm{v}$; glyphosate a $2.400,3.360 \mathrm{e}$ 4.320 g e.a. $\mathrm{ha}^{-1}$ com Silwet a $0,1 \% \mathrm{v} / \mathrm{v}$; 
imazapyr (Arsenal) a 750 e 1.500 g e.a. ha-1 e diquat (Reward) a 400 e 800 g i.a. ha ${ }^{-1}$ com aplicação seqüencial. Manteve-se uma testemunha sem aplicação de herbicidas. O delineamento experimental utilizado foi o inteiramente casualizado, com quatro repetições.

Os herbicidas foram aplicados três meses após o plantio, quando as plantas apresentavam altura de 45 e $65 \mathrm{~cm}$, para $B$. subquadripara e B. mutica, respectivamente. Aos sete dias após aplicação dos herbicidas, para B. subquadripara, e aos 10 dias, para $B$. mutica, foi realizada a aplicação seqüencial dos tratamentos com o herbicida diquat.

$\mathrm{Na}$ aplicação dos herbicidas foi utilizado um pulverizador costal, pressurizado por $\mathrm{CO}_{2}$ com pressão constante de 2 bars e equipado com um reservatório de 2 litros. O consumo de calda foi de 2001 ha $^{-1}$. A barra de aplicação foi equipada com dois bicos jato plano Teejet 110.02 XR, distanciados $50 \mathrm{~cm}$ entre si.

As condições climáticas no momento da aplicação dos herbicidas e da aplicação seqüencial dos tratamentos com o herbicida diquat estão apresentadas na Tabela 1. As parcelas foram protegidas, com lonas plásticas, de possíveis rajadas de vento no momento da aplicação.

As avaliações de controle das plantas daninhas foram visuais, por meio de uma escala percentual de notas, em que 0 (zero) corresponde a nenhuma injúria demonstrada pela planta e 100 (cem) corresponde à morte das plantas, segundo a Sociedade Brasileira da Ciência das Plantas Daninhas - SBCPD (1995). Essas avaliações foram realizadas aos 2, 4, 7, 9, 18, 37, 45, 53, 67 e 75 dias após aplicação dos herbicidas (DAA) para B. subquadripara e aos $2,4,8,10,16,24,32,40,51,67$ e 74 DAA para $B$. mutica. Ao final das avaliações visuais foi avaliada a massa seca da parte aérea das plantas.
Os parâmetros utilizados para o estabelecimento das notas foram: quantidade de plantas mortas, acúmulo de biomassa, inibição do crescimento, quantidade e uniformidade das injúrias e capacidade de rebrota das plantas.

Os resultados foram submetidos à análise de variância pelo teste $\mathrm{F}$ e as médias dos tratamentos foram comparadas pelo teste t a $5 \%$ de probabilidade, para os resultados da avaliação visual, e a $10 \%$ de probabilidade, para os resultados da avaliação da massa seca.

\section{RESULTADOS E DISCUSSÕES}

Na Tabela 2 estão apresentados os resultados das avaliações visuais de controle das plantas de $B$. subquadripara em diferentes épocas após a aplicação dos herbicidas.

Foi observado, já aos dois dias após a aplicação (DAA) dos herbicidas, que todas as plantas submetidas aos tratamentos com glyphosate apresentavam os primeiros sintomas de cloroses nas folhas. Esses sintomas foram crescentes para todas as doses até os 37 DAA, e o tratamento com a menor dose e acrescido de Aterbane apresentou controle menos eficiente que os demais. Entre essas avaliações os sintomas evoluíram para necroses.

A partir dos 37 DAA, plantas submetidas ao herbicida glyphosate apresentaram rebrotas, com exceção do tratamento com a dose de 4.320 g e.a. ha-1 com Silwet. Essas rebrotas foram mais severas e progressivas até os 75 DAA no tratamento com a menor concentração, independentemente do adjuvante, proporcionando com isso um controle apenas satisfatório. Nas outras doses, as rebrotas reduziram e o controle aumentou novamente, chegando a proporcionar controle excelente aos 75 DAA.

Tabela 1 - Condições climáticas no momento da aplicação dos herbicidas. Botucatu-SP, 2001

\begin{tabular}{|c|c|c|c|c|c|c|c|c|}
\hline \multirow{3}{*}{ Condição } & \multicolumn{4}{|c|}{ Aplicação dos herbicidas } & \multicolumn{4}{|c|}{ Reaplicação do diquat } \\
\hline & \multicolumn{2}{|c|}{$\begin{array}{c}\text { Brachiaria } \\
\text { subquadripara }\end{array}$} & \multicolumn{2}{|c|}{ Brachiaria mutica } & \multicolumn{2}{|c|}{$\begin{array}{c}\text { Brachiaria } \\
\text { subquadripara }\end{array}$} & \multicolumn{2}{|c|}{ Brachiaria mutica } \\
\hline & Inicial & Final & Inicial & Final & Inicial & Final & Inicial & Final \\
\hline Horário da aplicação & $9 \mathrm{~h} 45$ & $10 \mathrm{~h} 15$ & $10 \mathrm{~h} 20$ & $11 \mathrm{~h} 10$ & $12 \mathrm{~h} 25$ & $12 \mathrm{~h} 40$ & $18 \mathrm{~h} 10$ & $18 \mathrm{~h} 40$ \\
\hline Umidade relativa do ar $(\%)$ & 65 & 80 & 42 & 38 & 36 & 32 & 35 & 35 \\
\hline Temperatura do ar $\left({ }^{\circ} \mathrm{C}\right)$ & 24 & 22 & 28 & 30 & 30 & 33 & 30 & 30 \\
\hline
\end{tabular}


Também Furlani Jr. et al. (1997) avaliaram diferentes concentrações de glyphosate, em diferentes volumes de aplicação, para algumas plantas aquáticas em campo, entre elas B. subquadripara, e observaram bom controle proporcionado por este herbicida.

Verificou-se que o herbicida imazapyr apresentou controle bastante lento, sempre crescente, tendo como sintomas iniciais cloroses, seguidas de necroses, e que somente aos 53 DAA começaram a ocorrer diferenças entre as doses. Ao final do estudo - $75 \mathrm{DAA}$ - as duas concentrações mostraram-se eficientes no controle desta espécie.
O herbicida diquat proporcionou controle expressivo das plantas já aos 2 DAA, sendo este maior em sua dose mais alta. As plantas apresentavam suas folhas bastante danificadas, com sintomas de queima (necroses). Aos 7 DAA, as plantas apresentavam rebrotas, o que reduziu o controle. Apesar da reaplicação aos sete dias após a primeira aplicação, novas rebrotas surgiram, comprometendo o controle nas duas doses.

Na Tabela 3 estão apresentados os resultados das avaliações visuais de controle das plantas de $B$. mutica em diferentes épocas após a aplicação dos herbicidas.

Tabela 2 - Controle de Brachiaria subquadripara em diferentes épocas após a aplicação dos herbicidas. Botucatu-SP, 2001

\begin{tabular}{|c|c|c|c|c|c|c|c|c|c|c|c|}
\hline \multirow{2}{*}{ Tratamento } & \multirow{2}{*}{$\begin{array}{c}\text { Dose } \\
\left(\mathrm{g} \mathrm{i} / \mathrm{e} . \mathrm{a} \cdot \mathrm{ha}^{-1}\right)\end{array}$} & \multicolumn{10}{|c|}{ Dias Após Aplicação dos Herbicidas } \\
\hline & & 2 & 4 & 7 & 9 & 18 & 37 & 45 & 53 & 67 & 75 \\
\hline 1.Testemunha & - & - & - & - & - & - & - & - & - & - & - \\
\hline 2.glyphosate $+\mathrm{At}^{1^{1 /}}$ & 2.400 & $4,7 \mathrm{de}$ & $35,5 \mathrm{c}$ & $74,0 \mathrm{a}$ & $84,7 \mathrm{c}$ & $94,5 \mathrm{~b}$ & $98,2 \mathrm{a}$ & $96,0 \mathrm{c}$ & $93,2 \mathrm{c}$ & $92,5 \mathrm{c}$ & $92,2 \mathrm{~d}$ \\
\hline 3.glyphosate+At & 3.360 & $14,0 \mathrm{c}$ & $48,2 \mathrm{~b}$ & $78,5 \mathrm{a}$ & $90,7 \mathrm{ab}$ & $97,7 \mathrm{ab}$ & $99,0 \mathrm{a}$ & $97,7 \mathrm{abc}$ & $98,2 \mathrm{a}$ & $98,5 \mathrm{a}$ & $98,5 \mathrm{ab}$ \\
\hline 4.glyphosate+At & 4.320 & $10,5 \mathrm{~cd}$ & $54,0 \mathrm{~b}$ & $78,2 \mathrm{a}$ & $92,2 \mathrm{ab}$ & $98,5 \mathrm{ab}$ & $99,2 \mathrm{a}$ & $98,2 \mathrm{ab}$ & $99,2 \mathrm{a}$ & $99,2 \mathrm{a}$ & $99,2 \mathrm{ab}$ \\
\hline 5.glyphosate $+\mathrm{Sil}^{2 /}$ & 2.400 & $11,7 \mathrm{~cd}$ & $45,0 \mathrm{bc}$ & $77,5 \mathrm{a}$ & $88,2 \mathrm{bc}$ & $96,2 \mathrm{ab}$ & $98,7 \mathrm{a}$ & $96,5 \mathrm{bc}$ & $96,0 \mathrm{~b}$ & $95,0 \mathrm{~b}$ & $94,5 \mathrm{c}$ \\
\hline 6.glyphosate+Sil & 3.360 & $12,5 \mathrm{c}$ & $53,5 \mathrm{~b}$ & $73,5 \mathrm{a}$ & $89,7 \mathrm{ab}$ & $97,7 \mathrm{ab}$ & $99,0 \mathrm{a}$ & $98,5 \mathrm{ab}$ & $98,7 \mathrm{a}$ & 99,0 a & $99,2 \mathrm{ab}$ \\
\hline 7.glyphosate+Sil & 4.320 & $10,0 \mathrm{~cd}$ & $47,0 \mathrm{~b}$ & $79,0 \mathrm{a}$ & 93,7 a & $98,7 \mathrm{a}$ & $99,0 \mathrm{a}$ & $99,0 \mathrm{a}$ & $99,5 \mathrm{a}$ & $99,7 \mathrm{a}$ & $100,0 \mathrm{a}$ \\
\hline 8.imazapyr & 750 & $1,7 \mathrm{e}$ & $2,2 \mathrm{~d}$ & $4,2 \mathrm{c}$ & $7,2 \mathrm{e}$ & $42,2 \mathrm{c}$ & $83,0 \mathrm{c}$ & $87,2 \mathrm{~d}$ & $85,0 \mathrm{e}$ & $94,5 \mathrm{bc}$ & $95,5 \mathrm{c}$ \\
\hline 9.imazapyr & 1.500 & $0,0 \mathrm{e}$ & $1,2 \mathrm{~d}$ & $3,7 \mathrm{c}$ & $7,0 \mathrm{e}$ & $45,2 \mathrm{c}$ & $86,5 \mathrm{~b}$ & $87,2 \mathrm{~d}$ & $89,5 \mathrm{~d}$ & $97,7 \mathrm{a}$ & $98,0 \mathrm{~b}$ \\
\hline 10.diquat & 200 & $78,2 \mathrm{~b}$ & $77,7 \mathrm{a}$ & $64,0 \mathrm{~b}$ & $75,0 \mathrm{~d}$ & $43,7 \mathrm{c}$ & $25,5 \mathrm{~d}$ & $19,7 \mathrm{e}$ & $7,0 \mathrm{f}$ & $3,2 \mathrm{~d}$ & $2,2 \mathrm{e}$ \\
\hline 11.diquat & 400 & $86,5 \mathrm{a}$ & $81,2 \mathrm{a}$ & $65,2 \mathrm{~b}$ & $76,5 \mathrm{~d}$ & $43,2 \mathrm{c}$ & $23,0 \mathrm{e}$ & $19,5 \mathrm{e}$ & $7,7 \mathrm{f}$ & $3,7 \mathrm{~d}$ & $3,0 \mathrm{e}$ \\
\hline $\mathrm{F}$ tratamento & & $159,76^{* *}$ & $72,86^{* *}$ & $223,77 * *$ & $590,13 * *$ & $694,45^{* *}$ & $1921,45 * *$ & $3390,34 * *$ & $4705,94 * *$ & $3976,03 * *$ & $4604,46^{* *}$ \\
\hline CV (\%) & & 23,3 & 16,5 & 8,26 & 5,0 & 3,8 & 2,1 & 1,8 & 1,7 & 2,0 & 1,8 \\
\hline DMS & & 7,05 & 9,68 & 6,49 & 4,59 & 3,82 & 2,19 & 1,93 & 1,78 & 2,03 & 1,90 \\
\hline
\end{tabular}

Médias seguidas de mesma letra, na coluna, não diferem estatisticamente entre si pelo teste $\mathrm{t}(\mathrm{P}>0,05)$; ** valor significativo pelo teste $\mathrm{F}$ $\mathrm{a}(\mathrm{P}>0,01) ;{ }^{1 /} \mathrm{At}=$ Aterbane $0,5 \% \mathrm{v} / \mathrm{v} ; \stackrel{2}{ } / \mathrm{Sil}=$ Silwet $0,1 \% \mathrm{v} / \mathrm{v}$.

Tabela 3 - Controle de Brachiaria mutica em diferentes épocas após a aplicação dos herbicidas. Botucatu-SP, 2001

\begin{tabular}{|c|c|c|c|c|c|c|c|c|c|c|c|c|}
\hline \multirow{2}{*}{ Tratamento } & \multirow{2}{*}{$\begin{array}{c}\text { Dose } \\
\left(\mathrm{g} \mathrm{i} / \mathrm{e} . \mathrm{a} \cdot \mathrm{ha}^{-1}\right)\end{array}$} & \multicolumn{11}{|c|}{ Dias Após Aplicação dos Herbicidas } \\
\hline & & 2 & 4 & 8 & 10 & 16 & 24 & 32 & 40 & 51 & 67 & 74 \\
\hline 1. Testemunha & - & - & - & - & - & - & - & - & - & - & - & - \\
\hline 2. glyphosate $+\mathrm{At}^{\underline{1 /}}$ & 2.400 & $5,8 \mathrm{bc}$ & $42,0 \mathrm{~b}$ & $47,8 \mathrm{c}$ & $51,0 \mathrm{c}$ & $55,5 \mathrm{~d}$ & 74,3 bcd & $81,8 \mathrm{~b}$ & $92,8 \mathrm{bc}$ & $95,0 \mathrm{ab}$ & $95,3 \mathrm{bc}$ & 94,8 bc \\
\hline 3.glyphosate + At & 3.360 & $8,5 \mathrm{bc}$ & $43,5 \mathrm{~b}$ & $57,0 \mathrm{abc}$ & $63,0 \mathrm{abc}$ & $68,3 \mathrm{bcd}$ & $81,0 \mathrm{abc}$ & $90,0 \mathrm{a}$ & $96,8 \mathrm{ab}$ & 98,3 a & $98,8 \mathrm{ab}$ & $98,8 \mathrm{ab}$ \\
\hline 4.glyphosate+At & 4.320 & $7,5 \mathrm{bc}$ & $48,0 \mathrm{~b}$ & $56,5 \mathrm{abc}$ & $69,5 \mathrm{ab}$ & $74,5 \mathrm{abc}$ & $87,5 \mathrm{a}$ & $89,8 \mathrm{a}$ & $97,8 \mathrm{ab}$ & $98,0 \mathrm{a}$ & $98,3 \mathrm{ab}$ & $98,0 \mathrm{ab}$ \\
\hline 5.glyphosate $+\mathrm{Sil}^{2 !}$ & 2.400 & $11,3 \mathrm{~b}$ & $48,0 \mathrm{~b}$ & $56,0 \mathrm{bc}$ & $60,5 \mathrm{bc}$ & $63,3 \mathrm{~cd}$ & 70,5 cde & $81,8 \mathrm{~b}$ & $92,5 \mathrm{bc}$ & $94,5 \mathrm{ab}$ & $94,0 \mathrm{c}$ & $93,5 \mathrm{c}$ \\
\hline 6.glyphosate+Sil & 3.360 & $7,5 \mathrm{bc}$ & $45,0 \mathrm{~b}$ & $65,8 \mathrm{a}$ & $72,0 \mathrm{ab}$ & $73,3 \mathrm{abc}$ & $84,0 \mathrm{ab}$ & $91,5 \mathrm{a}$ & $96,5 \mathrm{ab}$ & $97,5 \mathrm{ab}$ & $98,0 \mathrm{ab}$ & $97,8 \mathrm{ab}$ \\
\hline 7.glyphosate+Sil & 4.320 & $7,8 \mathrm{bc}$ & $48,0 \mathrm{~b}$ & $62,0 \mathrm{ab}$ & $76,3 \mathrm{a}$ & $83,8 \mathrm{a}$ & $91,3 \mathrm{a}$ & $94,5 \mathrm{a}$ & $98,5 \mathrm{a}$ & $99,3 \mathrm{a}$ & $99,3 \mathrm{a}$ & $99,3 \mathrm{a}$ \\
\hline 8.imazapyr & 750 & $4,3 \mathrm{bc}$ & $7,5 \mathrm{c}$ & $13,5 \mathrm{~d}$ & $24,5 \mathrm{~d}$ & $24,3 \mathrm{e}$ & $60,0 \mathrm{e}$ & $74,8 \mathrm{c}$ & $84,8 \mathrm{~d}$ & $92,5 \mathrm{~b}$ & $99,5 \mathrm{a}$ & $99,8 \mathrm{a}$ \\
\hline 9.imazapyr & 1.500 & $2,3 \mathrm{c}$ & $6,3 \mathrm{c}$ & $17,3 \mathrm{~d}$ & $20,5 \mathrm{~d}$ & $30,0 \mathrm{e}$ & 69,3 de & $73,8 \mathrm{c}$ & $89,5 \mathrm{~cd}$ & $96,8 \mathrm{ab}$ & $100,0 \mathrm{a}$ & $100,0 \mathrm{a}$ \\
\hline 10.diquat & 200 & 54,8 a & $70,3 \mathrm{a}$ & $57,8 \mathrm{ab}$ & $34,0 \mathrm{~d}$ & $78,3 \mathrm{ab}$ & $29,8 \mathrm{f}$ & $9,3 \mathrm{~d}$ & $3,0 \mathrm{e}$ & $3,5 \mathrm{c}$ & $0,8 \mathrm{~d}$ & $0,8 \mathrm{~d}$ \\
\hline 11.diquat & 400 & $58,0 \mathrm{a}$ & $74,3 \mathrm{a}$ & $58,5 \mathrm{ab}$ & $35,0 \mathrm{~d}$ & $79,8 \mathrm{ab}$ & $27,5 \mathrm{f}$ & $7,0 \mathrm{~d}$ & $2,0 \mathrm{e}$ & $0,5 \mathrm{c}$ & $0,0 \mathrm{~d}$ & $0,0 \mathrm{~d}$ \\
\hline F tratamento & & $92,29 * *$ & $142,34 * *$ & $53,45^{* *}$ & $25,01 * *$ & $40,09 * *$ & $71,99 * *$ & $290,80 * *$ & $595,86^{* *}$ & $675,20 * *$ & $1499,36 * *$ & $1091,50 * *$ \\
\hline CV (\%) & & 26,3 & 10,5 & 14,0 & 21,6 & 15,0 & 11,3 & 7,0 & 5,1 & 4,8 & 3,3 & 3,9 \\
\hline DMS & & 6,14 & 5,98 & 9,06 & 14,33 & 12,47 & 10,04 & 6,38 & 5,10 & 4,94 & 3,40 & 3,98 \\
\hline
\end{tabular}

Médias seguidas de mesma letra, na coluna, não diferem estatisticamente entre si pelo teste $\mathrm{t}(\mathrm{P}>0,05)$; **, valor significativo pelo teste $\mathrm{F}$ $\mathrm{a}(\mathrm{P}>0,01) ;{ }^{1 /}$ At $=$ Aterbane $0,5 \% \mathrm{v} / \mathrm{v} ;{ }^{2}$ Sil $=$ Silwet $0,1 \% \mathrm{v} / \mathrm{v}$. 
Para o herbicida glyphosate, as plantas de B. mutica apresentaram os primeiros sintomas de injúrias aos 2 DAA, com início de clorose das folhas, independentemente das concentrações e dos adjuvantes.

Dos 8 aos 16 DAA os sintomas passaram a evoluir para necroses. Os tratamentos de 4.320 e 3.360 g e.a. ha ${ }^{-1}$ com Silwet e 4.320 g e.a. ha ${ }^{-1}$ com Aterbane apresentavam controle mais satisfatório, e as doses mais baixas proporcionavam menor controle das plantas.

Os sintomas aumentaram progressivamente para todos os tratamentos até os 51 DAA, com as concentrações de 3.360 e 4.320 g e.a. ha-1 apresentando sempre maior porcentagem de controle e não diferenciando entre si. Aos 51 DAA as plantas submetidas à dose mais baixa apresentaram sinais de rebrotas, o que prejudicou o tratamento, paralisando o controle até os 74 DAA.

Obien et al. (1973) testaram glyphosate para o controle de $B$. mutica - porém em dois estádios de desenvolvimento, sendo um em pleno desenvolvimento vegetativo e o outro no florescimento das plantas - e observaram controle de 71 e $80 \%$ para as doses de 2 e $4 \mathrm{~kg} \mathrm{ha}^{-1}$, respectivamente. As doses de 1 e $3 \mathrm{~kg} \mathrm{ha}^{-1}$ controlaram as plantas em desenvolvimento. Já Grassi \& Leiderman (1972), para as concentrações de 1 e $1,5 \mathrm{~kg} \mathrm{ha}^{-1}$, verificaram controle satisfatório de $B$. mutica com $40 \mathrm{~cm}$ de altura.

Com relação ao herbicida imazapyr, também aos 2 DAA as plantas exibiam seus primeiros sintomas, não apresentando diferenças entre as doses. A maior evolução do controle ocorreu a partir dos 20 DAA. Durante todo o período de avaliação não ocorreu diferença entre as duas doses (750 e 1.500 g e.a. ha-1), e aos 74 DAA ocorreu a morte das plantas na dose mais alta, apesar de a dose menor ter proporcionado controle muito semelhante.

O herbicida diquat já na primeira avaliação apresentou elevada porcentagem de controle; todas as folhas atingidas pelo herbicida apresentavam-se danificadas com queimaduras, não ocorrendo diferença entre as doses testadas. Aos 6 DAA as plantas começaram a

Tabela 4 - Massa seca das plantas de B. subquadripara e porcentagem de massa seca ao final do ensaio com relação à testemunha. Botucatu-SP, 2001

\begin{tabular}{|c|c|c|c|c|c|}
\hline \multirow{3}{*}{ Tratamento } & \multirow{3}{*}{$\begin{array}{c}\text { Dose } \\
\left(\mathrm{g} \mathrm{i} / \mathrm{e} . \mathrm{a} \cdot \mathrm{ha}^{-1}\right)\end{array}$} & \multicolumn{2}{|c|}{ B. subquadripara } & \multicolumn{2}{|c|}{ B. mutica } \\
\hline & & \multicolumn{2}{|c|}{ Massa seca } & \multicolumn{2}{|c|}{ Massa Seca } \\
\hline & & (g) & $(\%)$ & (g) & $(\%)$ \\
\hline 1. Testemunha & - & $1.305,6 \mathrm{a}$ & 100,0 & 707,8 a & 100,0 \\
\hline 2. glyphosate+Aterbane $0,5 \%$ & 2.400 & 297,7 de & 22,8 & $214,9 \mathrm{~d}$ & 30,4 \\
\hline 3. glyphosate+Aterbane $0,5 \%$ & 3.360 & 192,7 ef & 14,8 & $128,1 \mathrm{~d}$ & 18,1 \\
\hline 4. glyphosate+Aterbane $0,5 \%$ & 4.320 & 179,8 ef & 13,8 & $154,8 \mathrm{~d}$ & 21,9 \\
\hline 5. glyphosate+Silwet $0,1 \%$ & 2.400 & $240,5 \mathrm{ef}$ & 18,4 & $166,7 \mathrm{~d}$ & 23,6 \\
\hline 6. glyphosate+Silwet $0,1 \%$ & 3.360 & 182,4 ef & 14,0 & $125,6 \mathrm{~d}$ & 17,7 \\
\hline 7. glyphosate+Silwet $0,1 \%$ & 4.320 & $104,7 \mathrm{f}$ & 8,0 & $119,2 \mathrm{~d}$ & 16,8 \\
\hline 8. imazapyr & 750 & $417,3 \mathrm{~d}$ & 32,0 & $268,7 \mathrm{~cd}$ & 38,0 \\
\hline 9. imazapyr & 1.500 & 289,4 de & 22,2 & $177,8 \mathrm{~d}$ & 25,1 \\
\hline 10. diquat & 400 & $819,7 \mathrm{~b}$ & 62,8 & $420,6 \mathrm{bc}$ & 59,4 \\
\hline 11. diquat & 800 & $609,6 \mathrm{c}$ & 46,7 & $479,4 \mathrm{~b}$ & 67,7 \\
\hline $\mathrm{F}$ tratamento & & $25,03 * *$ & - & $6,07 * *$ & - \\
\hline $\mathrm{CV}(\%)$ & & 30,2 & - & 51,1 & - \\
\hline DMS & & 152,73 & - & 165,29 & - \\
\hline
\end{tabular}

Médias seguidas de mesma letra, na coluna, não diferem estatisticamente entre si pelo teste $\mathrm{t}(\mathrm{P}>0,1)$; **, valor significativo pelo teste $\mathrm{F}$ a $(\mathrm{P}>0,01)$. 
apresentar rebrotas também nas duas doses, e aos 10 DAA as plantas apresentavam o controle comprometido pelas rebrotas. Novamente, apesar da aplicação seqüencial aos dez dias após a primeira aplicação, novas rebrotas surgiram, inviabilizando o controle.

Na Tabela 4 estão apresentados os resultados da avaliação da massa seca das plantas de B. subquadripara e B. mutica ao final do experimento.

De maneira geral, os resultados obtidos para as duas espécies na avaliação da massa seca das plantas confirmam os resultados das avaliações visuais, apesar de as plantas submetidas ao herbicida imazapyr terem apresentado maior acúmulo de biomassa, comparadas às dos tratamentos com glyphosate, porém esse fato é atribuído ao controle mais rápido proporcionado pelo glyphosate e, conseqüentemente, à maior decomposição das plantas ao final do estudo.

\section{LITERATURA CITADA}

FURLANI JR., J. A. et al. Controle químico de macrófitas aquáticas emersas no reservatório de Barra Bonita-SP. In: CONGRESSO BRASILEIRO DA CIÊNCIA DAS PLANTAS DANINHAS, 21., 1.997, Caxambu. Resumos... Viçosa, MG: SBCPD, 1997. p. 330.

GRASSI, N.; LEIDERMAN, L. Controle de capim-angola (Panicum purpurascens Raddi) com herbicidas. In: SEMINÁRIO BRASILEIRO DE HERBICIDAS E ERVAS DANINHAS, 9., 1972, Campinas. Resumos... Campinas: 1972. p. 8.
KISSMANN, K. G. Plantas infestantes e nocivas. 2. ed. São Paulo: Basf, 1997. t. 1. 824 p.

LORENZI, H. Plantas daninhas do Brasil: terrestres, aquáticas, parasitas, tóxicas e medicinais. 2. ed. Nova Odessa: Instituto Plantarum, 1991. 440 p.

MARTINS, D. Controle de plantas daninhas aquáticas. In: WORKSHOP CONTROLE DE PLANTAS AQUÁTICAS, 1998, Brasília. Resumos... Brasília: IBAMA, 1998.

ROCHE, R.; MENÉNDEZ, J.; HERNÁNDEZ, J. E. Características morfológicas indispensables para la classificacion de espécies del gênero Brachiaria. Pastos Forrages, v. 13, p. 205-222, 1990.

OBIEN, S. R.; PLUCKNETT. D. L.; DELA PENA, R. S. Control of paragrass (Brachiaria mutica) with glyphosate and other herbicides. In: ASIAN-PACIFIC WEED SCIENCE SOCIETY CONFERENCE, 4., Rotorua. CAB Abstracts, 1972-1975. p. 518-522.

OLIVEIRA JR, R. S.; CONSTANTIN, J. Plantas daninhas e seu manejo. Guaíba: Agropecuária, 2001. 362 p.

PITELLI, R. A. Macrófitas aquáticas no Brasil, na condição de problemáticas. In: WORKSHOP CONTROLE DE PLANTAS AQUÁTICAS, 1998, Brasília. Resumos... Brasília: IBAMA, 1998. p. 12-15.

SOCIEDADE BRASILEIRA DA CIÊNCIA DAS PLANTAS DANINHAS - SBCPD. Procedimentos para instalação, avaliação e análise de experimentos com herbicidas. Londrina: SBCPD, 1995. 42 p.

TANAKA, R. H. Prejuízos provocados pelas plantas aquáticas. In: WORKSHOP CONTROLE DE PLANTAS AQUÁTICAS, 1998, Brasília. Resumos... Brasília: IBAMA, 1998. p.36-38. 